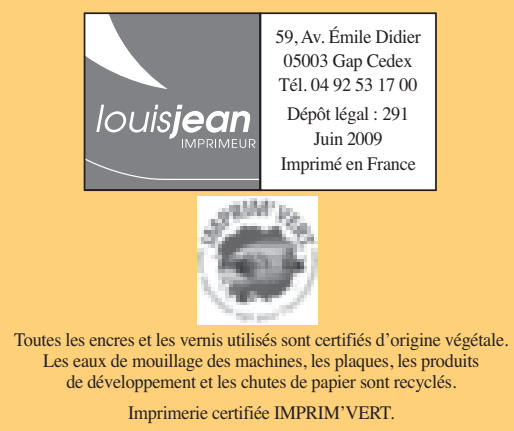

0
0
0
0
0
0
0
0
0
0
0
0
0
0
0
0
7
0
0

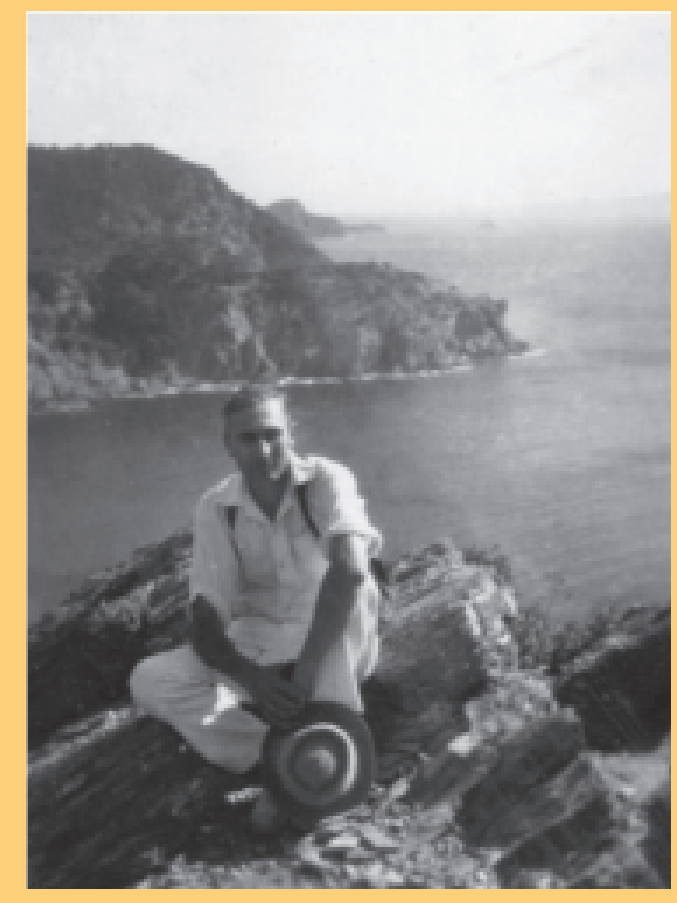

Diffusion

De Boccard 


\section{SOMMAIRE}

Avant-propos, par A. Hermary, Président du Centre ................................................. 7

In memoriam Paul Åström 1929-2008 (A. CAubet, R. Merrillees) .............................. 9

In memoriam Nicolas Coldstream 1928-2008 (V. Karageorghis, R. Merrillees) .... 13

\section{DOSSIER J. BÉRARD}

\section{L’archéologie à Chypre dans les années 1950}

Antoine HERMARY, Introduction :

1. Les recherches de Jean Bérard à Chypre (1950-1953) :

la correspondance, archives du Département des Antiquités

de Chypre et de l'École Française de Rome

2. Franz Georg MAIER, Archaeology in Cyprus in the Fifties :

Jean Bérard's work at Nea Paphos in context

3. Robert MerrilleEs, Cyriote Antiquities from Nicosia-Ayia Paraskevi

acquired by the Melbourne Cyprus Expedition in 1955/56

4. Jean BÉRARD (article inédit), La colonisation grecque de Chypre et la date de la Guerre de Troie

5. Sabine Fourrier, Légendes de fondation et hellénisation de Chypre.

Parcours historiographique 103

\section{ÉTUDES}

Lone W. Sørensen, An “odd fellow”

Béatrice Blandin, Thierry Petit, Isabelle Tassignon,

Recherches récentes au palais d'Amathonte

Laurence ALPE, Les tombes d'enfants à Chypre à l'époque des royaumes :

bilan et perspectives

Lucie Bonato, Le consulat de France à Larnaca à la fin de la Monarchie de Juillet.

Correspondance de Dagobert Fourcade et Théodore Goepp (1840-1849).

IV : La Justice. Les affaires du Sieur Georges Diab

Louis LAPIERRE, Georges Lapierre (1789-1846), Seigneur de Chypre et proscrit 


\section{CHRONIQUE}

Michel Amandry, Chronique numismatique chypriote (IV)

\section{COMPTES RENDUS D'OUVRAGES}

1. Sabine FOURRIER, La coroplastie chypriote archaïque. Identités culturelles et politiques à l'époque des royaumes, Lyon, 2007 [A. Hermary]

2. Franz-Georg Maier, contributions de S. Hadjisavvas et M.-L. von Wartburg, Führer durch Alt-Paphos (Kouklia), Nicosie, 2006 [A. Hermary]

3. Near Eastern Archaeology, vol. 71:1-2, March-June 2008. Ancient Cyprus: American Research [A. Hermary]

\section{INDEX}

INDEX des Cahiers 27-36, 1997-2006, établi par Lucie Bonato 
DOSSIER J. BÉRARD

\title{
5. LÉGENDES DE FONDATION ET HELLÉNISATION DE CHYPRE Parcours historiographique
}

\section{Sabine FOURRIER}

\begin{abstract}
This article presents a review of studies devoted to the problem of the hellenization of Cyprus in the Late Bronze Age, which were published since Bérard's unpublished work about the foundation legends in the fifties.
\end{abstract}

La recherche de la part de vérité historique qui se dissimule derrière les textes légendaires a été au cœur des travaux de Jean Bérard, et elle est à l'origine de son activité archéologique à Chypre. C'est, en effet, dans le but de retrouver la cité fondée par Agapénor qu'il entreprit des fouilles à Kato Paphos, puis dans la nécropole de Ktima ${ }^{1}$. Son article, probablement rédigé en 1953-1954, est resté inédit et il n'a donc pas pu nourrir les travaux ultérieurs ${ }^{2}$. Peu d'entre eux ont porté spécifiquement sur les Nostoi eux-mêmes ${ }^{3}$, mais ces derniers apparaissent, de manière plus ou moins explicite, dans d'innombrables écrits consacrés à la fin du Bronze Récent à Chypre. De fait, l'archéologie de cette période a connu un développement sans précédent depuis les années cinquante, grâce à la découverte et à la fouille de nombreux sites, mais aussi grâce à la publication

1. S. J. Bérard, «Introduction », dans J. Deshayes, La nécropole de Ktima, Paris, 1963, p. 9-14.

2. V. Karageorghis, Chypre, Genève, 1968, p. 67 : «Le très regretté Jean Bérard qui avait fouillé dans la région de Paphos, avait essayé de résoudre les problèmes liés à la légende de fondation de Paphos par Agapénor, roi de Tégée. ».

3. Depuis l'article de Gjerstad 1944, auquel répond J. Bérard, on peut mentionner Fortin 1980 et 1984, Vanschoonwinkel 1991, p. 293-312, et, dans une perspective essentiellement méthodologique, Baurain 1989. 
de nouvelles études concernant différentes catégories de matériel, et notamment la céramique.

Proposer un parcours historiographique représente donc sans aucun doute une gageure. Le sujet a suscité une immense bibliographie, qui comprend de nombreux articles, des colloques, des publications de fouilles, des synthèses par période chronologique ou par type de matériel, mais aussi des ouvrages de vulgarisation. Il est impossible de rendre compte de toutes les contributions, de faire une place à tous les auteurs qui ont participé au débat, et il est inévitable, en tentant de dégager des évolutions, de distinguer des étapes et, somme toute, des progrès, de recourir à certaines simplifications.

À la fin du Bronze Récent se situe un basculement majeur, qui fait que la civilisation chypriote de l'Âge du Fer est de langue majoritairement, mais non pas exclusivement, grecque, alors qu'elle ne l'était pas auparavant. Certes, les enseignements de l'archéologie post-coloniale invitent à ne pas sous-estimer le poids du contexte contemporain, celui dans lequel s'inscrit la recherche et qui a vu se constituer un État chypriote dans un douloureux et difficile débat identitaire ${ }^{4}$. Mais c'est faire peu de cas de la qualité et de la richesse de la plupart des travaux que de les réduire à l'affirmation, plus ou moins consciente, de thèses nationalistes. L'hellénisation de Chypre - au sens linguistique - n'est pas plus une invention contemporaine que les « Étéo-Chypriotes » ${ }^{5}$. Si le fait est là, le processus reste difficile à saisir. Je propose un parcours historiographique, non pas une prise de position dans un débat toujours en cours, et encore moins une solution à un problème dont on peut être sûr, et c'est tant mieux, qu'il alimentera encore de nombreux travaux. De fait, les questions suscitées dépassent largement le cadre d'un débat chypro-centré, elles touchent aux méthodes de l'histoire ancienne elles-mêmes. Les documents sont rares, et l'on ne

4. V. Karageorghis, « The Crisis Years: Cyprus », dans W. A. Ward, M. Sharp Joukowsky (éds), The Crisis Years: the 12th Century B.C., Dubuque, 1992, p. 82 : "The present-day "Greekness" of Cyprus had somehow to be explained against a historical-archaeological background. This need has been felt even more strongly in recent years since Cyprus has been going through a crucial period in asserting her identity. This coincidence was unfortunate as the emotional factor (for or against) was not always easy to eliminate. »; et, plus récemment, «Being a Cypriot, I often find myself in an embarrassing position, always running the risk of being influenced, or of being accused of having been influenced in my scholarship by present-day polities in Cyprus. » (Karageorghis 2000 , p. 255). Le poids de la formation et du milieu explique, selon N. Leriou, le point de vue hellénocentriste adopté par les chercheurs qui, du XIX siècle à Vassos Karageorghis, ont construit la théorie de l'hellénisation de Chypre («Constructing an Archaeological Narrative: the Hellenization of Cyprus », Stanford Journal of Archaeology, accessible en ligne sur le site www.stanford.edu/ dept/archaeology/journal). Je n'ai pas pu consulter deux autres de ses articles : « The Mycenaean Colonisation of Cyprus under the Magnifying Glass », dans G. Muskett, A. Koltsida, M. Georgiadis (éds), SOMA 2001: Symposium on Mediterranean Archaeology, Oxford, 2002, p. 169-177, et "Locating Identities in the Eastern Mediterranean : the Case of "Hellenised Cyprus" », dans S. Antoniadou, A. Pace (éds), Mediterranean Crossroads, Athènes-Oxford, 2007, p. 563-591.

5. Voir M. Given, « Inventing the Eteocypriots: Imperialist Archaeology and the Manipulation of Ethnic Identity », JMA 11, 1998, p. 3-29, et la réponse de Th. Petit, «Eteocypriot Myth and Amathusian Reality », JMA 12, 1999, p. 108-120. 
peut pas se permettre d'en rejeter purement et simplement une catégorie au prétexte que textes, archéologie et histoire événementielle constituent souvent des sources divergentes, parfois un impossible ménage à trois. Dans ces débats, la terminologie est lourde de sens : que l'on désigne les acteurs comme «Peuples de la mer » ${ }^{6}$, « Mycéniens », «Achéens » ou «Grecs », comme « réfugiés », « conquérants » ou « migrants », le processus comme une « colonisation ${ }^{7}$, une « hellénisation », une « pénétration » ou une « hybridation ${ }^{8}$, on n'entend pas la même chose, et il ne s'agit pas de nuances. Mais les apports de cette immense bibliographie ne sont pas seulement méthodologiques. Si certaines positions paraissent irréconciliables, le débat est loin d'avoir été stérile. Certes, la fin du Bronze Récent à Chypre offre aujourd'hui une image beaucoup plus complexe que celle qu'on en avait il y a cinquante ans, mais il suffit justement de comparer ce fossé épistémologique avec le calme plat des recherches concernant l'Âge du Fer, pour lequel la synthèse d'E. Gjerstad, pourtant datée de 1948, n'est toujours pas dépassée, pour mesurer l'étendue des progrès accomplis.

\section{Jusqu'au début des années soixante : une question de date}

L'article qu'E. Gjerstad consacre aux légendes de fondation ${ }^{9}$ remet radicalement en cause l'idée d'une colonisation mycénienne de l'île à date haute, qui prévalait alors dans le monde de l'archéologie chypriote ${ }^{10}$. En effet, pour plusieurs savants, en particulier Cl. Schaeffer, le fouilleur d'Enkomi, de véritables communautés, colonies ou emporia, étaient établies à Chypre dès le XIV ${ }^{\mathrm{e}}$ siècle, et elles étaient notamment responsables de la diffusion dans l'île de céramique mycénienne ${ }^{11}$. C'était aussi l'hypothèse défendue par J. Bérard, pour qui l'hellénisation de Chypre ne pouvait pas être le fait de réfugiés fuyant l'effondrement du système palatial sur le continent, mais bien celui d'une civilisation mycénienne conquérante, alors à son apogée ${ }^{12}$.

Pour E. Gjerstad, s'il n'est pas question de nier l'existence d'un épisode migratoire dans l'histoire de Chypre au Bronze Récent, ce dernier n'a pas pu avoir lieu dès le $X V^{e}$ siècle. Il propose de distinguer deux vagues de «colonisation» achéenne, la première au début du $\mathrm{XII}^{\mathrm{e}}$ siècle (dont se ferait l'écho la légende de Teucros), la seconde

6. Auxquels il faut ajouter les « Structural Sea Peoples » de Sherratt 1998.

7. Voir les remarques introductives de Iacovou 2008.

8. Voskos, Knapp 2008.

9. Gjerstad 1944.

10. Voir le résumé proposé par Baurain 1989, p. 464-465.

11. Cl. Schaffer, Enkomi-Alasia I, Paris, 1952.

12. Voir Schaeffer 1973, p. 287 : «Well, they must have been very wealthy refugees, to come to build such a splendid town as that of Enkomi-Alasia. » Le même argument réapparait, dans un schéma tout autre, mais qui vise de la même façon à infirmer l'hypothèse d'une migration massive d'Achéens à Chypre au début du XII ${ }^{\mathrm{e}}$ siècle, lorsque $\mathrm{S}$. Sherratt décrit ce mouvement comme allant «from the periphery to the core, from the Provinces to Versailles » (Sherratt 1992, p. 325). 
vers 1100 av. J.-C. (elle serait reflétée par les autres légendes jugées recevables). Ce schéma est d'autant plus remarquable que peu de sites du Bronze Récent étaient connus avant guerre : outre les travaux de la mission française à Enkomi, E. Gjerstad avait luimême fouillé une forteresse à Nitovikla, et l'Université de Pennsylvanie menait des recherches à Episkopi-Bamboula. La reconstitution proposée par E. Gjerstad réussit à emporter l'adhésion de plusieurs chercheurs, au premier rang desquels il faut placer A. Furumark ${ }^{13}$. Surtout, elle parut recevoir une confirmation éclatante de l'archéologie qui, essentiellement à partir des années soixante, connut un essor prodigieux, notamment grâce à l'action de V. Karageorghis. Lorsqu'en 1972, Cl. Schaeffer tire les conclusions du colloque «The Mycenaeans in the Eastern Mediterranean», dont il traduit fort justement le sujet en «Coming of the Greeks to Cyprus », il est le dernier à porter la voix des «Anciens » (Myres, Sjöqvist ${ }^{14}$ ), contre les « Modernes » (Desborough, Catling, Cadogan, Karageorghis), désormais majoritaires ${ }^{15}$. Plus personne aujourd'hui ne défend la thèse d'une colonisation mycénienne aux XIV et XIII ${ }^{\mathrm{e}}$ siècles. Si C. Baurain propose de situer au cours du Chypriote Récent II les premiers moments, décisifs, de l'hellénisation de l'île ${ }^{16}$, dont les effets durables n'auraient été visibles que des siècles plus tard, à l'orée de l'Âge du Fer, c'est au terme d'un raisonnement tout autre, et dans un schéma où il n'est plus question ni de colonisation ni d'emporia.

\section{Des années soixante au milieu des années quatre-vingt : une colonisation en deux vagues migratoires}

Le développement de l'archéologie chypriote depuis la deuxième guerre mondiale a entraîné la fouille de nombreux sites du Bronze Récent et suscité des études qui ont paru confirmer, à la fois l'impossibilité d'une colonisation mycénienne de l'île aux XIV XIII ${ }^{\mathrm{e}}$ siècles, et l'existence de deux phases migratoires à la fin du Bronze Récent. Ces dernières pouvaient être situées précisément, à la charnière du Chypriote Récent IICChypriote Récent IIIA pour la première, au début du Chypriote Récent IIIB pour la seconde. L'archéologie prouvait le bien-fondé d'une hypothèse, elle donnait raison à E. Gjerstad.

13. A. Furumark, « The Excavations at Sinda. Some Historical Results », OpAth 6, 1965, p. 108110.

14. La position d'E. Sjöqvist est, en réalité, sensiblement différente, puisqu'il réfute l'hypothèse d'une colonisation achéenne au Chypriote Récent II, tout en interprétant Enkomi comme un emporion « levanto-helladique » (Problems of the Late Cypriote Bronze Age, Stockholm, 1940).

15. Schaeffer 1973.

16. Baurain 1989. Des Mycéniens se seraient installés à Chypre, à titre individuel, au cours des XIV et XIII 'siècles. L'effondrement d'Alashiya, vers 1200 av. J.-C., aurait entraîné un bouleversement de la structure politique et sociale de l'île et permis à ces populations de langue grecque, présentes à Chypre depuis plusieurs siècles, de prendre progressivement le pouvoir. 
Les indices concordants s'accumulaient au fur et à mesure des progrès sur le terrain. La connaissance topographique de l'île au Bronze Récent a été complétée par l'exploration de Sinda, d'Apliki et de Myrtou-Pigadhes au lendemain de la guerre, par celle de Kouklia/Palaepaphos à partir de 1950, puis de Kition à partir de 1959. Les chantiers de fouilles se sont multipliés après l'indépendance de Chypre : Hala Sultan Tekke, Maroni, Kalavasos, Athienou, Phlamoudi, Pyla-Kokkinokremos, Maa-Palaekastro, Alassa. Tous les sites qui présentaient une occupation continue au cours des $\mathrm{XIV}^{\mathrm{e}}$-XIII ${ }^{\mathrm{e}}$ siècles livraient de la céramique mycénienne, mais en quantités plus faibles que les céramiques locales, réalisées sans l'aide du tour, Base-Ring et White Slip. Par ailleurs, l'étude de cette céramique, confortée par des analyses physico-chimiques, a révélé qu'à l'exception de quelques séries, qui apparaissent dans la seconde moitié du XIII ${ }^{\mathrm{e}}$ siècle, elle était fabriquée dans des ateliers péloponnésiens ${ }^{17}$. Chypre appartenait donc bien à la koinè culturelle qui caractérisait alors la Méditerranée orientale, elle participait sans doute activement à la diffusion de cette civilisation matérielle «internationale»- les signes chypro-minoens gravés sur des céramiques de type mycénien trouvées hors de l'île le suggèrent -, mais elle ne faisait pas partie du monde mycénien ${ }^{18}$.

Il en allait tout autrement pour la fin du Bronze Récent. La transition entre le Chypriote Récent IIC et le Chypriote Récent IIIAétait marquée sur le terrain par une série d'abandons ou de destructions : certains sites importants, comme Kalavasos ou Maroni, qui avaient été fondés au Chypriote Récent IIC, étaient abandonnés à la fin de la période ; d'autres, comme Enkomi ou Kition, étaient entièrement remodelés. Par ailleurs, des établissements d'un type nouveau, à vocation défensive et d'occupation brève (Pyla-Kokkinokremos, Maa-Palaekastro) témoignaient de conditions instables. Enfin, la civilisation matérielle de Chypre au XII ${ }^{\mathrm{e}}$ siècle paraissait complètement différente de celle de la période précédente. Les fabriques de tradition chypriote, modelées, disparaissaient au profit de céramiques de type égéen, réalisées sur le tour, qui étaient produites localement ${ }^{19}$. Une nouvelle fabrique modelée (Barbarian ou Handmade Burnished Ware), attestée en Grèce, lui était parfois associée ${ }^{20}$. Les innovations étaient nombreuses dans le domaine du bâti (fortifications de type cyclopéen, aménagement de foyers centraux, baignoires), du religieux (cornes de consécration) ou de la métallurgie (épées de type Naue II, jambières, fibules), autant de traits qui paraissaient provenir du monde égéen ${ }^{21}$.

17. Catling 1986, p. 598 : «This (...) affects ideas about 14th and 13th cent. Aegean colonies or emporia in Cyprus, reducing their credibility to vanishing point. ».

18. Pour une vue synthétique de la période : L. Steel, Cyprus before History, Londres, 2004, p. $149-186$.

19. Catling 1986, p. 608.

20. V. Karageorghis, "Barbarian Ware" in Cyprus », dans V. Karageorghis (éd.), Cyprus between the Orient and the Occident, Nicosie, 1986, p. 246-258.

21. Pour un relevé exhaustif, voir Karageorghis 2000. 
Une nouvelle rupture était sensible à la fin du Chypriote Récent IIIA. À l'exception de Kition et de Palaepaphos, tous les sites de l'Âge du Bronze étaient définitivement abandonnés au profit de sites «neufs », comme Salamine. Une nouvelle céramique (Proto-White Painted), au répertoire d'inspiration mycénienne, était utilisée dans toutes les régions de l'île. Elle accompagnait les défunts dans un nouveau type de tombe à chambre, à dromos long et étroit, d'origine égéenne. Enfin, la découverte dans une tombe de Palaepaphos-Skales d'un obélos inscrit en syllabaire, mais en grec, achevait de trahir l'origine ethnique des migrants.

Tous les faisceaux paraissaient convergents, l'archéologie confirmait les textes, elle illustrait les événements ${ }^{22}$. Deux vagues achéennes avaient touché Chypre, une première fois au moment de la destruction des palais mycéniens, dans la série de mouvements de population connus des sources égyptiennes sous le nom de «Peuples de la mer», puis à la fin du XII et au début du XI ${ }^{\mathrm{e}}$ siècle, cette deuxième «colonisation », qui allait donner naissance à la plupart des villes-capitales de royaumes chypriotes de l'Âge du Fer, étant évoquée dans les légendes de fondation. Le colloque de Nicosie en 1972 («The Mycenaeans in the Eastern Mediterranean ») consacrait le triomphe de cette thèse. La communication de F.G. Maier y est exemplaire du positivisme alors dominant: le matériel archéologique (c'est-à-dire essentiellement la céramique) y est agencé de façon à documenter une interprétation considérée comme acquise ; non seulement peut-on distinguer, grâce à la fouille, deux moments d'installation des Grecs à Palaepaphos, mais on peut même suivre leurs pas depuis l'avant-poste fortifié de Maa jusqu'à la ville de Kinyras ${ }^{23}$. Cette tendance a eu ses excès, ainsi dans deux articles de M. Fortin, où toute découverte archéologique datable du Chypriote Récent vient confirmer la véracité de toutes les légendes de fondation, ces dernières n'étant jamais examinées, contrairement à la démarche d'E. Gjerstad, dans leur contexte de production ${ }^{24}$. Rares sont les auteurs qui, à cette époque, expriment une position plus nuancée, hésitant à lire dans le sol les témoignages indiscutables d'une colonisation mycénienne. C'est le cas, par exemple, d'H. Catling, qui, lors du colloque de 1972, sans remettre en question l'existence de migrations, souligne les continuités de la civilisation chypriote du Bronze Récent et les apports multiples qui façonnent l'identité de celle de l'Âge du Fer :

22. Karageorghis 1984, p. 19: «For this period we have written documents and therefore we cannot - and should not - avoid the temptation to correlate archaeological and historical events. »

23. F.G. Maier, «Evidence for Mycenaean Settlement at Old Paphos », dans The Mycenaeans in the Eastern Mediterranean, Nicosie, 1973, p. 68-78. L'exposé est construit en trois points : 1. « Evidence for a first wave of immigrants », 2. «Evidence for a second wave of Greek settlers », 3. «The route of the settlers ».

24. Fortin 1980 et 1984. En introduction au second article, il résume sa démarche comme suit : «J'y réunissais les preuves archéologiques qui tendent à démontrer la véracité des légendes entourant la fondation, à la fin de l'Âge du Bronze Récent, de certaines cités importantes de Chypre (...) par des héros grecs au retour de la guerre de Troie. » (1984, p. 133). 
«What remained at the end? A handful of centres of settlement - Salamis, Kition, Kourion, Palaepaphos, Lapithos chief amongst them, enjoying a material civilisation whose origins owed at least as much to the dying Bronze Age world of Crete, of continental Greece and of Syria-Palestine as to its own Cypriot background. (...) It [la « colonisation » mycénienne de l'île] should be understood as a process of joining forces with people of differing cultures, and of living with them largely as equals rather than as dominating them; intermarrying, rather than segregating. (...) One of the strangest phenomena of this process is the absence of evidence for burial according to the Aegean rite - only in the very last years of Late Cypriot IIIB, it seems, were chamber tombs of Aegean type introduced to Cyprus. Equally, to my mind, the abundant evidence for religious activity revealed at Enkomi, Kition and Myrtou is of a kind uncharacteristic of the Mycenaean world. (...) We may easily multiply the facets of the Late Cypriot III period which stand apart from the activity of the full Mycenaean world - but we can just as easily multiply the elements in Late Cypriot III for which we have little or no Cypriot precedent. We see the end products of a process of amalgamation in which Cypriot, Aegean and Near Eastern cultures were brought together to produce not a mixture but a compound ${ }^{25}$. ».

On remarquera que les implications chronologiques de cette reconstitution des événements n'ont guère été prises en considération, alors qu'elles sont au cœur de l'article de J. Bérard. S'il y avait bien une colonisation égéenne, qui prouvait la véracité des légendes, quelle était la phase migratoire que ces dernières documentaient ? Toutes les légendes, à l'exception de celle relative à Kourion, situent ces fondations dans le cadre des Nostoi, des retours de héros achéens au lendemain de la guerre de Troie ${ }^{26}$. Le titre de l'article inachevé de J. Bérard le montre bien : si la colonisation grecque de Chypre a lieu, comme il le pense, au XIV siècle, c'est aussi à cette époque que l'on doit situer la guerre de Troie ${ }^{27}$. Selon E. Gjerstad, seule la légende de Teucros « 1'Anatolien » pouvait correspondre à la première vague migratoire, les autres, celles du moins qui avaient un fondement historique, se rapportaient à la seconde. Le corpus légendaire rassemblait

25. H. Catling, «The Achaean Settlement of Cyprus », dans The Mycenaeans in the Eastern Mediterranean, Nicosie, 1973, p. 38-39. L' « hybridization » de Voskos, Knapp 2008, n'est qu'un terme moins élégant pour le « compound», défini par H. Catling il y a plus de trente ans.

26. Cf. Baurain 1989, p. 467 : «Peut-on considérer la guerre de Troie comme appartenant au domaine de l'histoire événementielle, c'est-à-dire comme un fait intrinsèque et qui est donc susceptible d'être situé de manière formelle sur l'échelle du temps qui passe ? Faut-il préciser que c'est seulement dans le cas d'une réponse affirmative que l'on serait alors autorisé à aborder l'étape suivante de la réflexion, la deuxième question : quelle valeur reconnaître aux divers récits de fondation impliquant des centres chypriotes et qui tous - à la seule exception de Kourion situent ces installations par rapport à la guerre de Troie ?»

27. S. J. Bérard, «Introduction », dans J. Deshayes, La nécropole de Ktima, Paris, 1963, p. 9 , n. 1 : «C'était pour lui un argument qui, joint à beaucoup d'autres, lui permettait d'assurer que ce $\mathrm{XIV}^{\mathrm{e}} \mathrm{s}$. où la civilisation mycénienne conquérante apparaissait en pleine expansion était aussi le siècle de la Guerre de Troie. » 
ainsi en un seul moment deux mouvements de population distincts de près d'un siècle. Les fondations étaient dissociées de leur événement déclencheur, avec lequel elles n'entretenaient plus qu'un vague rapport de postériorité. Les progrès de l'archéologie ont montré qu'aucune des villes évoquées dans les légendes, à l'exception de Palaepaphos, n'est occupée avant le Chypriote Récent IIIB, c'est-à-dire près d'un siècle après la date qu'Ératosthène attribue à la guerre. La plupart des chercheurs ont donc naturellement associé toutes les légendes de fondation avec la seconde vague migratoire, malgré l'apparent hiatus chronologique ${ }^{28}$. La découverte de niveaux du $\mathrm{XI}^{\mathrm{e}}$ siècle à Salamine confirmait cette hypothèse. Quant à Palaepaphos, qui existe déjà au Chypriote Récent II, elle n'était pas fondée, mais refondée par Agapénor. Seul M. Fortin, dans un premier article soucieux d'établir l'entière véracité des textes, a soutenu que les légendes évoquaient toutes la première phase de colonisation achéenne : si le matériel le plus ancien découvert à Salamine datait du $\mathrm{XI}^{\mathrm{e}}$ siècle, c'est que les fouilleurs n'avaient pas atteint les niveaux de la ville de Teucros ${ }^{29}$. Toutes les fondations de villes grecques étaient datables de ca. 1200 av. J.-C., c'est-à-dire de l'époque où l'on situe traditionnellement la guerre de Troie. Dans un article suivant, toutefois, il se rallie à l'opinion commune ${ }^{30}$. De manière amusante, l'argument chronologique apparaît pourtant au détour des travaux pour prouver que la fondation de Teucros ne peut pas être localisée sur le site de Salamine, qui n'est occupé que bien après les « événements », mais qu'il s'agit d'une refondation d'Enkomi, « PalaeSalamine $»^{31}$ : on retrouve ainsi, sous une autre forme, l'hypothèse d'E. Gjerstad, qui dissocie des autres la légende salaminienne ${ }^{32}$.

\section{Depuis le milieu des années quatre-vingt : une colonisation invisible ?}

Démontrée un temps par l'archéologie, la thèse d'une colonisation achéenne de Chypre fut bientôt démontée par les mêmes moyens. Les critiques portèrent sur les méthodes employées, notamment la pertinence des témoignages archéologiques pour échafauder des reconstitutions historiques. Certes, des destructions et des abandons ont lieu à la fin du Chypriote Récent IIC et au début du Chypriote Récent IIIA, mais tous ces événements sont-ils contemporains et découlent-ils de la même cause ? Certes, une

28. Voir, par exemple, V. Karageorghis, Chypre, Genève, 1968, p. 66-67.

29. Fortin 1980, p. 43.

30. Fortin 1984, p. 144.

31. Par exemple, à la suite de P. Dikaios (Enkomi II, Mayence, 1971, p. 519-521), M. Yon, «La fondation de Salamine », dans Salamine de Chypre. Histoire et archéologie, Paris, 1980, p. 79.

32. J. Vanschoonwinkel, «La présence grecque à Chypre au XI siècle av. J.-C. », dans V. Karageorghis (éd.), Cyprus in the 11th Century B.C., Nicosie, 1994, p. 125-126 : « Si le noyau authentique de la légende de fondation de Salamine par Teucros évoque vraisemblablement la reconstruction d'Enkomi au début du XII ${ }^{\mathrm{e}}$ siècle, en revanche les autres fondations légendaires peuvent seulement être datées, sans autre précision, de la période postérieure à la guerre de Troie, c'est-à-dire de la fin du II ${ }^{\mathrm{e}}$ millénaire ou même plus tard. » 
nouvelle céramique, de type mycénien, est produite localement au Chypriote Récent IIIA, mais les vases sont-ils des marqueurs ethniques, susceptibles de révéler la migration de nouvelles populations ? Après le temps des enthousiasmes, le temps de la réflexion révélait la fragilité de beaucoup d'hypothèses qui, s'étayant les unes les autres, avaient fini par bâtir un château de cartes ${ }^{33}$.

Les progrès sur le terrain montraient que les ruptures qui marquaient la transition entre le Chypriote Récent IIC et le Chypriote Récent IIIA étaient loin d'être partout semblables ni même évidentes. Certains établissements étaient définitivement abandonnés, sans destruction, à la fin du Chypriote Récent IIC ou au début de la période suivante (Maroni, Kalavasos) ; d'autres étaient détruits puis reconstruits (Enkomi) ; d'autres enfin étaient remodelés et connaissaient un développement urbain remarquable sans porter trace de la moindre destruction (Kition). On assistait donc certes à des changements, des changements sans doute rapides même s'ils n'étaient vraisemblablement pas tous exactement contemporains, mais il devenait difficile de proposer une même cause pour tant d'effets, un même événement pour tant d'histoires ${ }^{34}$.

Par ailleurs, s'il y avait des abandons, il n'y avait pas de fondation nouvelle, à l'exception de Maa-Palaekastro et Pyla-Kokkinokremos, qui ne furent occupés que brièvement pendant la période de «crise » ${ }^{35}$. L'interprétation des sites eux-mêmes était remise en question. Ainsi, les « avant-postes » fortifiés, notamment celui de MaaPalaekastro, livraient un matériel comparable à celui des autres habitats contemporains de l'île : si donc le site abritait des colons, lors de leurs premiers pas dans la conquête de Chypre, ces derniers n'y étaient en tout cas pas seuls et ils n'étaient peut-être pas si conquérants que cela ${ }^{36}$. Enfin, les traits nouveaux qui apparaissaient au Chypriote Récent IIIA ne révélaient plus nécessairement la présence de nouvelles populations et ils n'étaient pas forcément d'origine égéenne ${ }^{37}$.

Quant à l'ensemble de la civilisation matérielle de Chypre au XII ${ }^{\mathrm{e}}$ siècle, il y manquait certains éléments caractéristiques de la civilisation mycénienne, comme les tombes à tholos ou les archives en linéaire B. Il en allait de même pour la céramique, considérée

33. Le revirement le plus spectaculaire est celui de F. G. Maier, « Kinyras und Agapenor », dans V. Karageorghis (éd.), Cyprus between the Orient and the Occident, Nicosie, 1986, p. 311-320. Voir également Sherratt 1992.

34. Voir le résumé de Iacovou 2007, p. 465-466 : «Multiple Settlement Histories ».

35. M. Iacovou, « Society and Settlements in Late Cypriot III », dans E. Peltenburg (éd.), Early Society in Cyprus, Édimbourg, 1989, p. 52-59.

36. Karageorghis 1990, p. 26 : "It is true, however, that the material culture at Maa is not significantly different from the local Cypriote material evidence. (...) Considering these points we may now elaborate on the proposal made earlier and suggest that at Maa there was either a joint entreprise between Cypriots and Mycenaeans, or that the settlement of the Mycenaeans was sanctioned by the Cypriots, who may have had strong military and mercantile inducements to accept them. »

37. Pour tous les éléments de la civilisation matérielle, voir le résumé de Voskos, Knapp 2008. 
jusqu'alors comme le marqueur par excellence. La fabrique Myc.IIIC:1b était déjà attestée dans des niveaux du Chypriote Récent IIC, donc antérieurs aux destructions et à l'arrivée de populations mycéniennes dont elle était censée trahir la présence. La comparaison des faciès céramiques de Chypre et du continent grec révélait que les destructions des palais mycéniens étaient bien antérieures aux destructions/abandons chypriotes et que les deux événements n'étaient pas, du moins dans leur chronologie, directement liés ${ }^{38}$. Par ailleurs, la céramique chypriote de type mycénien témoigne d'influences et d'inspirations de diverses régions égéennes et elle entretient des rapports étroits avec les autres fabriques décorées contemporaines, fabriquées sur le tour, qu'il conviendrait de rassembler sous une même appellation, sans référent ethnique « White Painted Wheelmade III » ${ }^{39}$.

Si la migration devenait invisible, si l'on pouvait se passer de l'hypothèse d'une arrivée de populations égéennes ${ }^{40}$, comment rendre compte des changements, indéniables ? S. Sherratt a insisté sur l'importance des facteurs sociaux et politiques internes qui, considérés sur le long terme, permettent d'envisager le Chypriote Récent IIIA non pas comme une ère nouvelle, mais comme un développement, une suite logique du Chypriote Récent II. La céramique, jusqu'alors considérée comme le champ des ruptures, témoignerait, au contraire, de continuités, de fluidités : les productions du XII ${ }^{e}$ siècle, tournées, sont les produits d'une industrie, contrôlée par un pouvoir centralisé, à la différence des fabriques chypriotes traditionnelles, modelées, qu'elles finissent par évincer. Elles sont la preuve que Chypre profite de l'effondrement des systèmes économiques «impériaux » à la fin du XIII ${ }^{\mathrm{e}}$ siècle. C'est l'aboutissement de la participation de l'île à un réseau de commerce international, marquée sur le terrain par le développement de centres côtiers. Cette nouvelle économie, ouverte, exacerbe la concurrence entre les nouveaux centres : les destructions, déplacements, abandons, sont les conséquences de crises internes, de «Peuples de la mer structurels ${ }^{41}$.

38. E. French, P. Åström, «A Colloquium on Late Cypriote III Sites », RDAC 1980, p. 267-269.

39. Voir B. Kling, Mycenaean IIIC:1b and Related Pottery in Cyprus (SIMA 87), Göteborg, 1989 ; ead., « Mycenaean IIIC:1b and Related Pottery in Cyprus: Comments on the Current State of Research », dans E. Oren (éd.), The Sea Peoples and their World: a Reassessment, Philadelphie, 2000, p. 281-295 (avec un bilan historiographique); Sherratt 1991. Le terme a été forgé par P. Åström, The Swedish Cyprus Expedition IV/1C, Lund, 1972, p. 276-289.

40. On notera toutefois que les tenants de cette théorie du développement interne (contre l'hypothèse de changements dus à un facteur extérieur), et en particulier S. Sherratt, ne vont toutefois jamais jusqu'à nier la réalité d'une arrivée et d'une installation durable de populations égéennes à Chypre. Leur critique porte sur les méthodes de l'interprétation, non pas sur l'interprétation elle-même. Par exemple, Sherratt 1991, p. 195: «Finally it ought to be stressed that the type of interpretative framework presented here need not necessarily be thought of as incompatible with the idea of people of Aegean origin living and operating in Cyprus throughout the LCII and IIIA periods, or even with the idea of some relatively large scale immigration at some point within this timespan.»

41. Sherratt 1998. 
Les critiques, parfois radicales, adressées à la théorie "traditionnelle », héritée d'E. Gjerstad, ne sont pas restées sans effet sur les « Modernes » devenus «Anciens » ${ }^{42}$. L'aménagement le plus important a porté sur la chronologie. La première phase de migration n'est plus considérée comme un mouvement massif, mais découpée en une série de vagues successives : dès la seconde moitié du XIII ${ }^{\mathrm{e}}$ siècle, vers 1230 av. J.-C., de nouvelles populations arrivent à Chypre et s'installent dans des sites provisoires (Maa-Palaekastro et Pyla-Kokkinokremos). Vers 1200, une nouvelle vague atteint l'île (destruction et reconstruction de Maa, abandon de Pyla) ${ }^{43}$. Une nouvelle subdivision de la période (Chypriote Récent IIC:2) permet de rendre compte de l'apparition de céramique Myc.IIIC:1b produite localement, avant le $\mathrm{XII}^{\mathrm{e}}$ siècle ${ }^{44}$. Les migrants ne sont plus exclusivement égéens, mais ils comprennent des Anatoliens et des ProcheOrientaux. Ils ne viennent plus en conquérants, habitant des établissements séparés, mais ils coexistent avec des Chypriotes ${ }^{45}$. La colonisation achéenne de l'île est toutefois visible, les changements sensibles dans la civilisation chypriote du XII ${ }^{\mathrm{e}}$ siècle restent dus à l'arrivée et à l'installation durable de populations et non pas seulement à des évolutions internes. La théorie des deux vagues migratoires proposée par E. Gjerstad demeure ainsi valide, même s'il ne s'agit plus de deux mouvements massifs, mais de deux temps forts dans une pénétration plus longue et plus diffuse ${ }^{46}$.

La déconstruction de l'hypothèse d'une colonisation achéenne de Chypre vers 1200 av. J.-C. a eu un autre effet, majeur : l'accent, autrefois mis essentiellement sur le $\mathrm{XII}^{\mathrm{e}}$ siècle, a été repoussé sur la seconde vague migratoire, au $\mathrm{XI}^{\mathrm{e}}$ siècle. Cette évolution

42. Voir les remarques réflexives de V. Karageorghis, "The Crisis Years: Cyprus », dans W. A. Ward, M. Sharp Joukowsky (éds), The Crisis Years: the 12th Century B.C. from beyond the Danube to the Tigris, Dubuque, 1992, p. 82: «Not very long ago, after the discoveries at Enkomi and Sinda and the final publication of Enkomi by P. Dikaios, we thought that a new world in Cypriot archaeology had emerged which would explain the later mythical and literary sources about "the Achaean colonization of Cyprus". We thought (note the we) that the vast amounts of Mycenaean IIIC:1b pottery, unearthed for the first time at Enkomi and Sinda, would provide ample archaeological justification for the identity of the peoples who appeared on the Cypriot horizon after ca. 1200 B.C. as the predecessors of Teucer and Agapenor. »

43. Karageorghis 1984 ; V. Karageorghis, M. Demas, Pyla-Kokkinokremos. A Late 13th Century B.C. Fortified Settlement in Cyprus, Nicosie, 1984, p. 66-75.

44. V. Karageorghis, M. Demas, Excavations at Kition V. The Pre-Phoenician Levels, Nicosie, 1985, p. 263-280.

45. Voir les conclusions très prudentes, au regard de ce qui avait été écrit auparavant, de V. Karageorghis, M. Demas, Excavations at Maa-Palaekastro 1979-1986, Nicosie, 1988, p. 264 : "The intimation of co-existence leads to the third theory - namely, that Maa represents a joint enterprise between Cypriots and "group X" peoples, or perhaps that the settlement of "group X" was sanctioned by Cypriots. »

46. Voir la vue d'ensemble proposée par V. Karageorghis, Early Cyprus. Crossroads of the Mediterranean, Los Angeles, 2002, p. 71-141. 
de pensée, sensible dès le milieu des années quatre-vingt ${ }^{47}$, connaît sa consécration lors du colloque de Nicosie sur le $\mathrm{XI}^{\mathrm{e}}$ siècle ${ }^{48}$. Contrairement à la précédente, cette hypothèse, pourtant tout aussi fragile, n'a guère été contestée. La raison en a souvent été soulignée par M. Iacovou : les spécialistes de l'Âge du Bronze chypriote ne sont pas les mêmes que ceux de l'Âge du Fer, le Chypriote Récent IIIB est trop tardif pour les premiers, trop ancien pour les seconds ; la période est confinée aux marges des études, elle ne trouve pas sa place dans l'histoire de l'île ${ }^{49}$. Au cours de nombreux articles, M. Iacovou a progressivement développé une vue originale, qui tente de rendre compte de l'hellénisation de Chypre et de ses effets sur la longue durée, sans forcer la documentation archéologique. Au XII siècle, la migration est invisible ; au $\mathrm{XI}^{\mathrm{e}}$ siècle, elle est achevée puisque la civilisation matérielle de l'île témoigne d'une remarquable homogénéité ${ }^{50}$ et que sa topographie est déjà, dans ses grandes lignes, celle de l'Âge du Fer ${ }^{51}$.

Comment rendre compte, dans ces conditions, du processus d'hellénisation ? Dans un article récent, I. Voskos et B. Knapp contournent l'aporie, en suggérant que les transformations que connaît Chypre à la fin du Bronze Récent sont dus à une lente « hybridation », mais ils maintiennent l'hypothèse de l'installation de « quelques personnes d'origine égéenne », « à un moment au cours du XI ${ }^{\mathrm{e}}$ siècle » ${ }^{52}$. Toutefois, si la civilisation chypriote $\mathrm{du} \mathrm{XI}^{\mathrm{e}}$ siècle témoigne bien d'une création originale, la langue ne porte aucune trace de «créolisation». De fait, l'argument linguistique est au cœur des travaux de M. Iacovou ${ }^{53}$, mais il n'est pas le seul et il est intimement lié à des arguments d'ordre politique et social, envisagés sur la longue durée. L'introduction de la langue grecque à Chypre n'a pu avoir lieu qu'au XII ${ }^{\mathrm{e}}$ siècle, quand des populations égéennes illettrées (le linéaire $\mathrm{B}$, réservé à une élite administrative, disparaît avec les palais mycéniens)

47. Par exemple, V. Karageorghis, M. Demas, Excavations at Kition V. The Pre-Phoenician Levels, Nicosie, 1985, p. 276 : «The complete hellenization of Cyprus, with the introduction of the Greek language, the Mycenaean funerary architecture etc., is a feature of the 11th c. B.C. (...) The traditional view, therefore, to which many of us suscribed for a number of years, that from the very beginning of LCIIIA the island was colonized by Achaeans who became the new rulers, should thus be modified. This colonization must have been a slow process, perhaps resulting from the continuous arrival of colonists and their progressive political ascent. »

48. V. Karageorghis (éd.), Cyprus in the 11th Century B.C., Nicosie, 1994.

49. Une «phobie» qui touche d'ailleurs l'ensemble de la période géométrique: voir, par exemple, Iacovou 1999.

50. Iacovou 2005. Pour la céramique, voir notamment M. Iacovou, «Proto-White Painted Pottery: a Classification of the Ware », dans J. A. Barlow, D. L. Bolger (éds), Cypriot Ceramics: Reading the Prehistoric Record, Philadelphie, 1991, p. 199-205: "Its appearance marks the coming together of all the earlier painted wares of LCIIIA. PWP could not have been suddenly imported to Cyprus by newcomers; it was the result of local amalgamation. »

51. M. Iacovou, « The Topography of Eleventh Century B.C. Cyprus », dans V. Karageorghis (éd.), Cyprus in the 11th Century B.C., Nicosie, 1994, p. 149-165 ; Iacovou 1999.

52. Voskos, Knapp 2008, p. 679.

53. Iacovou 2005. 
apprennent à transcrire leur langue dans le syllabaire local, alors toujours employé ${ }^{54}$. Ces immigrants ne s'installent pas dans des enclaves, ils n'arrivent pas avec un « bagage culturel » complet ${ }^{55}$, même s'ils sont certainement responsables de certaines nouveautés dans la civilisation matérielle de l'île. Ils ne sont pas des réfugiés mais appartiennent à une élite, dont les chefs portent le titre mycénien de basileis ${ }^{56}$. Ils n'imposent pas un système politique de type mycénien, mais ils vont progressivement prendre le pouvoir dans les différents centres, politiquement autonomes, de l'île ${ }^{57}$. Invisible quand elle se produit, $\mathrm{au} \mathrm{XII}^{\mathrm{e}}$ siècle, la migration devient visible dans ses effets, au XI ${ }^{\mathrm{e}}$. Si M. Iacovou maintient donc l'hypothèse de deux phases dans l'hellénisation de Chypre, seule la première, au $\mathrm{XII}^{\mathrm{e}}$ siècle, correspond à une migration; la seconde est celle de la prise du pouvoir, et c'est à cette dernière que les légendes de fondation font écho ${ }^{58}$. Cette reconstitution de la protohistoire chypriote, qui repose sur une vision longue de l'histoire de l'île, a des implications, en amont comme en aval. Elle suppose, tout d'abord, que la structure socioéconomique de l'île, du moins au Chypriote Récent IIC, est fragmentée en un certain nombre de centres autonomes, qu'il n'existe pas de royaume centralisé d'Alashiya, dont l'effondrement entraînerait une rupture dans l'organisation politique et sociale ${ }^{59}$. Elle place, par ailleurs, la naissance des royaumes chypriotes de l'Âge du Fer au XI ${ }^{\mathrm{e}}$ siècle ${ }^{60}$. L'hellénisation de l'île est indissociable du système politique de la royauté.

«Bien malin qui pourra démontrer dans quelle mesure la reconstitution de l'hellénisation de Chypre proposée ici est assez proche de la réalité. » ${ }^{61} \mathrm{Il}$ est, de fait, difficile de passer de l'observation archéologique à la reconstitution de phénomènes historiques, et le cheminement est trop souvent inverse : on recherche dans le sol

54. M. Iacovou, «"Greeks", "Phoenicians” and "Eteocypriots”. Ethnic Identities in the Cypriote Kingdoms », dans "Sweet Land..." Lectures on the History and Culture of Cyprus, Camberley, 2006, p. 36-39.

55. La prolifération, de courte durée, de tombes à fosse (shaft graves) au XII siècle pourrait toutefois permettre de saisir la présence de ces immigrants, invisibles dans l'habitat parce qu'ils s'intègrent dans une civilisation urbaine, mais visibles dans les tombes, parce qu'ils représentent des individus isolés et non pas des groupes familiaux : Iacovou 2008, p. 232.

56. M. Iacovou, « From the Mycenaean QA-SI-RE-U to the Cypriote PA-SI-LE-WO-SE: the Basileus in the kingdoms of Cyprus », dans S. Deger-Jalkotzy, I. S. Lemos (éds), Ancient Greece: From the Mycenaean Palaces to the Age of Homer, Édimbourg, 2005, p. 315-335.

57. Iacovou 2007.

58. M. Iacovou, «Cultural and Political Configurations in Iron Age Cyprus: The Sequel to a Protohistoric Episode », AJA 112, 2008, p. 648 : «It is the second phase that the foundation legends justify by referring to the ascendance of Greeks in specific polities to a position of authority. »

59. Contrairement à l'idée d'un royaume disparaissant brutalement vers 1200, développée par Cl. Baurain, Chypre et la Méditerranée orientale au Bronze Récent (ÉtChypr VI), Paris, 1984.

60. Contrairement à l'hypothèse d'une formation étatique tardive, notamment défendue par D. Rupp (par exemple, Rupp 1998).

61. Baurain 1989, p. 473. 
l'illustration d'événements, connus par d'autres sources. Mais le recours à des modèles théoriques ${ }^{62}$ force tout autant la documentation que la lecture au pied de la lettre des textes légendaires. Malgré l'absence de consensus, les débats qui portent sur la fin du Bronze Récent et l'hellénisation de Chypre sont loin de se résumer à un dialogue de sourds. Ils sont, par ailleurs, riches d'enseignements pour l'étude d'autres périodes de l'histoire de l'île. L'exemple le plus évident est celui de la « colonisation phénicienne », et il n'est sans doute pas étonnant qu'E. Gjerstad y ait consacré un article ${ }^{63}$, ni que M. Iacovou, dans sa conception de la longue durée, $\mathrm{y}$ fasse fréquemment référence ${ }^{64}$. Dès les $\mathrm{XI}^{\mathrm{e}}-\mathrm{X}^{\mathrm{e}}$ siècles av. J.-C., des céramiques phéniciennes sont importées à Chypre et elles suscitent, dès cette époque, des imitations locales. Faut-il supposer que des Phéniciens sont déjà installés dans l'île, dans des « colonies » ou des « emporia »? Dans les niveaux «phéniciens » les plus anciens de Kition-Kathari, dans les nécropoles géométriques de la ville, la migration est invisible. Ce n'est qu'à partir de l'époque archaïque, et plus encore à l'époque classique, que la civilisation matérielle de Kition présente des traits culturels phéniciens, mais la migration a déjà eu lieu, la civilisation matérielle de Kition est déjà « chyprophénicienne ». Là encore, à l'Âge du Fer comme à l'Âge du Bronze, on n'appréhende que le terme du processus, et non pas ses étapes. Si, en effet, la « colonisation » achéenne de Chypre au Bronze Récent n'a rien à voir avec la colonisation grecque en Sicile ${ }^{65}$, Kition n'a rien à voir non plus avec Motyé. Doit-on, pour autant, rejeter les textes qui évoquent une colonie tyrienne?

Dans les études consacrées à l'hellénisation de Chypre, les légendes de fondation ont occupé une place mineure, au bénéfice de l'archéologie. Elles ont, le plus souvent, servi à confirmer la réalité d'un événement, prouvé ou remis en question par les documents archéologiques ${ }^{66}$. Peu de travaux ont porté sur le contexte de production de ces légendes elles-mêmes, dont les plus anciens témoignages datent du $\mathrm{v}^{\mathrm{e}}$ siècle, sur les raisons de leur élaboration. E. Gjerstad avait pris soin de démêler le vrai du faux, et insisté sur les motifs idéologiques qui sous-tendent un certain nombre de textes ${ }^{67}$. Cl. Baurain a poussé la critique à son terme, considérant que les légendes sont les produits d'érudits hellénistiques qui, pétris d'Homère, savaient que l'île au moment de la guerre de Troie était la terre de Kinyras, mais se devaient de rendre compte qu'à leur époque, et depuis

62. Voskos, Knapp 2008.

63. E. Gjerstad, « The Phoenician Colonization and Expansion in Cyprus », RDAC 1979, p. 230254.

64. Iacovou 1999, 2008.

65. Cl. Baurain, Les Grecs et la Méditerranée orientale, Paris, 1997, p. 143.

66. Par exemple, H. W. Catling, The Cambridge Ancient History II-2 ( $3^{e}$ édition), Cambridge, 1975, p. 215-216 : "Since there was no comparable movement in the Iron Age, whatever historical worth these traditions hold belongs to the Bronze Age. »

67. Gjerstad 1944. Sa position est reprise par Vanschoonwinkel 1991, p. 293-312. 
longtemps, elle parlait grec ${ }^{68}$. C'est toutefois oublier que ces légendes sont avant tout le produit des Chypriotes eux-mêmes, et plus précisément de l'élite de l'île, c'est-à-dire de ses rois. C'est ce qu'a bien vu D. Rupp lorsqu'il lie ces ascendances légendaires à la mise en scène de la royauté archaïque ${ }^{69}$. Mais cela n'implique pas que les royaumes chypriotes, qui seraient alors de création récente, aient eu besoin d'asseoir leur légitimité sur un passé mythique. De fait, il est possible de trouver un écho de ces légendes bien plus tôt, de déceler la mise en scène d'un passé achéen dès le $\mathrm{XI}^{\mathrm{e}}$ siècle. C'est ce qu'a montré S. Sherratt, en étudiant deux des rares scènes figurées connues sur des vases Proto-White Painted ${ }^{70}$. Comme elle le souligne, l'attirail des personnages représentés fait référence à des symboles égéens, dont certains, tel le bouclier en forme de 8 , sont déjà des antiquités à l'époque où ils sont peints. Ce référent légendaire, qui représente l'un des fondements de l'idéologie royale chypriote, semble donc déjà présent lors de la phase de formation des royaumes. Il réapparaît lors de moments importants de leur longue vie, notamment à l'époque archaïque, puis à l'époque classique quand Chypre, devant l'empire assyrien puis lors du conflit gréco-perse, doit définir son identité. On ajoutera que cette identité n'est pas seulement achéenne. Kinyras est associé à Teucros et Agapénor, et pas seulement sur un mode négatif, parce que les nouveaux venus achéens chassent les « autochtones ». Sur la base de sa statue, érigée dans un sanctuaire de la « Paphienne » à Nicosie, Nicoclès se proclame descendant de Kinyras ${ }^{71}$. Le contexte joue certainement : prêtre de la Grande Déesse, le roi de Paphos se pose ainsi à tout le moins en protecteur du sanctuaire de Lédra. Mais cette référence à un personnage légendaire, qui régnait sur toute l'île de Chypre $^{72}$, n'est sans doute pas innocente à une époque où les rois chypriotes sont animés d'ambitions hégémoniques.

Université Lyon 2-HiSoMA, CNRS (UMR 5189), Lyon

\section{BIBLIOGRAPHIE}

BAURAin (Cl.), 1989, « Passé légendaire, archéologie et réalité historique : l'hellénisation de Chypre », Annales Économies Sociétés Civilisations 44, p. 463-477.
Catling (H. W.), 1986, «Archaeological Comment », dans R.E. Jones, Greek and Cypriot Pottery. A Review of Scientific Studies, Athènes, p. 574-609.

68. Baurain 1989, p. 473 : « En définitive, Agapénor, Teukros et les autres offrent autant de sujets d'étude pour le chercheur qui souhaite pénétrer les réactions mentales des anciens Grecs en quête de leur passé historique, mais les héros fondateurs resteront autant de leurres pour l'archéologue qui cherche à faire parler de vieilles pierres désespérément muettes. »

69. Rupp 1998. De même, A. Hermary a mis en évidence la présence de références héroïques dans l'iconographie archaïque: «Les ascendances légendaires des rois chypriotes : quelques messages iconographiques », CCEC 32, 2002 (Hommage à Marguerite Yon), p. 275-288.

70. Sherratt 1992.

71. SEG XX, 114.

72. Cl. Baurain, « Kinyras. La fin de l'Âge du Bronze à Chypre et la tradition antique », $B C H$ 104, 1980, p. 277-308. 
FORTIN (M.), 1980, «Fondation de villes grecques à Chypre : légendes et découvertes archéologiques », dans J.-B. Caron, M. Fortin, G. Maloney (éds), Mélanges d'études anciennes offerts à Maurice Lebel, Québec, p. 25-44.

ForTin (M.), 1984, « Nouvelles découvertes relatives aux légendes de fondation de villes grecques à Chypre à la fin de l'Âge du Bronze », Échos du monde classiquel Classical Views 3, p. 133-146.

Gjerstad (E.), 1944, «The Colonization of Cyprus in Greek Legend», OpArch 3, p. 107-123.

IAcovou (M.), 1999, «Excerpta Cypria Geometrica. Materials for a History of Geometric Cyprus », dans M. Iacovou, D. Michaelides (éds), Cyprus. The Historicity of the Geometric Horizon, Nicosie, p. 141166.

IAcovou (M.), 2005, «Cyprus at the Dawn of the First Millenium BC: Cultural Homogenisation versus the Tyranny of Ethnic Identifications », dans J. Clarke (éd.), Archaeological Perspectives on the Transmission and Transformation of Culture in the Eastern Mediterranean, Oxford, p. 125-134.

IAcovou (M.), 2007, «Advocating Cyprocentricism: An Indigenous Model for the Emergence of State Formation on Cyprus », dans A. Ben-Tor et alii, "Up to the Gates of Ekron". Essays in Honor of Seymour Gitin, Jérusalem, p. 461-475.

IAcovou (M.), 2008, « Cyprus: from Migration to Hellenisation », dans G.R. Tsetskhladze (éd.), Greek Colonisation. An Account of Greek Colonies and Other Settlements Overseas, Leyde-Boston, p. 219-288.

Karageorghis (V.), 1984, « New Light on Late Bronze Age Cyprus », dans V. Karageorghis, J.D. Muhly (éds), Cyprus at the Close of the Late Bronze Age, Nicosie, p. 19-22.
KARAgEORGhis (V.), 1990, The End of the Late Bronze Age in Cyprus, Nicosie.

KARAgEORGHIS (V.), 2000, « Cultural Innovations in Cyprus Relating to the Sea Peoples", dans E. Oren (éd.), The Sea Peoples and their World: a Reassessment, Philadelphie, 2000, p. 255-279.

Rupp (D.W.), 1998, « The Seven Kings of the Land of Ia', a District on Ia-ad-na-na:Achaean Bluebloods, Cypriot Parvenus or Both?», dans K.J. Hartswick, M.C. Sturgeon (éds), Studies in Honor of Brunilde S. Ridgway, Philadelphie, 1998, p. 209-222.

SCHAEFFER (Cl.), 1973, «Remarks and Conclusions », dans The Mycenaeans in the Eastern Mediterranean, Nicosie, p. 285-289.

Sherratt (S.), 1991, « Cypriot Pottery of Aegean Type in LCII-III: Problems of Classification, Chronology, and Interpretation ", dans J.A. Barlow， D.L. Bolger， B. Kling (éds), Cypriot Ceramics: Reading the Prehistoric Record, Philadelphie, 1991, p. 185-198.

SHERratt (S.), 1992, «Immigration and Archaeology: Some Indirect Reflections », dans P. Åström (éd.), Acta Cypria 2, Jonsered, p. 316-347.

Sherratt (S.), 1998, «Sea Peoples and the Economic Structure of the Late Second Millenium in the Eastern Mediterranean ", dans S. Gitin, A. Mazar, E. Stern (éds), Mediterranean Peoples in Transition, Jérusalem, p. 292-313.

VAnschoonwinkel (J.), 1991, L'Égée et la Méditerranée orientale à la fin $d u$ II ${ }^{e}$ millénaire, Louvain-Providence.

Voskos (I.), Knapp (A.B.), 2008, « Cyprus at the End of the Late Bronze Age: Crisis and Colonization or Continuity and Hybridization? », AJA 112, p. 659-684. 